\title{
Use of Appraisals of DisAbility Primary and Secondary Scale-Short Form (ADAPSS-sf) in individuals with pediatric-onset spinal cord injury
}

\author{
Kyle C. Deane ${ }^{1} \cdot$ Kathleen M. Chlan ${ }^{1} \cdot$ Lawrence C. Vogel $^{1,2} \cdot$ Kathy Zebracki $^{1,3}$
}

Received: 14 June 2019 / Revised: 18 October 2019 / Accepted: 21 October 2019 / Published online: 7 November 2019

(c) The Author(s), under exclusive licence to International Spinal Cord Society 2019

\begin{abstract}
Objectives To investigate the psychometric properties and utility of the Appraisals of DisAbility Primary and Secondary Scale-Short Form (ADAPSS-sf), a measure of cognitive appraisals, among adults with pediatric-onset SCI. To examine the relation of the ADAPSS-sf to demographics, injury characteristics, and secondary health and psychosocial outcomes.

Study design A structured telephone interview was conducted to obtain measures of ADAPSS-sf, pain, sleep, secondary health complications, and psychosocial functioning.

Setting Community in United States and Canada.

Participants Individuals who sustained an SCI at 18 years of age or younger $(N=115)$ were initially interviewed at age 19 years or older and followed annually.

Results Study findings support sound psychometrics of the ADAPSS-sf. The measure demonstrated strong test-retest reliability and internal consistency. There were no differences on ADAPSS-sf scores in relation to current age, gender, race, etiology, injury severity, or injury level. Individuals who sustained SCI at an older age were more likely to endorse negative appraisals of their injury. Results suggest that higher negative SCI-related appraisals were related to higher mental health difficulties. Negative SCI-related appraisals were associated with sleep difficulties, pressure injuries, pain, distress from pain, and poor overall subjective ratings of health.

Conclusions This study confirms the use of the ADAPSS-sf in a pediatric-onset SCI adult population by demonstrating its good internal validity, test-retest reliability, convergent and face validity, and brevity. Moreover, the current study revealed that such appraisals are associated with both psychosocial and secondary health outcomes, further supporting the ADAPSSsf as a valuable tool for clinicians and researchers.
\end{abstract}

\section{Introduction}

Spinal cord injury (SCI) is a life-altering neurologic disorder that can result in significant physical and psychosocial challenges. Pediatric-onset SCI, which represents $\sim 20 \%$ of SCIs [1] presents a unique set of challenges as children and adolescents live and cope with the impact of their SCI for a longer period of time compared to adult-onset SCI, requiring

Kathy Zebracki

kzebracki@shrinenet.org

Shriners Hospitals for Children, Chicago, IL, USA

Rush Medical College, Chicago, IL, USA

3 Northwestern University Feinberg School of Medicine, Chicago, IL, USA continuous adjustment throughout the lifespan. SCI acquired in childhood has been associated with a variety of secondary health conditions, including pressure injuries, urinary tract infections, scoliosis, sleep disturbances, pain, and negative subjective ratings of health [2,3]. Moreover, associations have been found between pediatric-onset SCI and a number of negative psychosocial outcomes, including increased anxiety and depressive symptoms $[4,5]$, as well as reduced satisfaction with life [6].

Despite these potential deleterious outcomes, individuals with SCI also demonstrate resiliency [7]. Injury characteristics, such as severity or level of injury, are generally weak predictors of subsequent functioning and emotional wellbeing [8], supporting the important role of psychosocial factors during recovery. An individual's cognitive appraisal of his or her disability has emerged as an important, and potentially causal, predictor of recovery [9-12]. Cognitive 
appraisals of a stressful event involve the initial characterization of the event, as well as judgements about appropriate responses [11]. According to the transactional stress appraisal and coping model [13], individuals estimate the possible impact of the stressor (primary appraisal), categorizing it as a loss, threat, or challenge. Subsequently, individuals make estimations about potential responses, available coping resources, and potential for success (secondary appraisals) [14]. Cognitive appraisals of injury predict psychological outcomes equal to or better than coping and functional independence $[9,15]$, suggesting that appraisals may be an important focus of clinical intervention following SCI.

In order to develop a measure of cognitive appraisals specifically for adults with SCI, Dean and Kennedy [16] developed the Appraisals of DisAbility: Primary and Secondary Scale (ADAPSS). This initial validation study revealed that SCI-related appraisals predicted depression within their adult sample, suggesting that the ADAPSS was a useful measure for assessing clinical needs and predicting adjustment within this population. A short form of the ADAPSS (ADAPSS-sf) was described by the authors but psychometric properties were not presented within this initial study. Three later studies have examined its clinical utility within two veteran samples [10, 12], and within an acutely injured adult sample [17] revealing sound psychometric properties, a two-factor structure (Catastrophic Negativity and Determined Resilience), and strong correlations with life satisfaction and depression. The literature supports the use of the ADAPSS-sf as a valuable tool in measuring the effects of the cognitive appraisals on psychosocial adjustment in adult-onset SCI; however, this measure has not been tested in the pediatriconset population. The pediatric-onset SCI population may differ in response from adult-onset given that children and adolescents learn to adjust to life with SCI across developmental periods and may develop other mechanisms of adaptation.

The goal of the current study was to assess the psychometric properties and clinical utility of the ADAPSS-sf among adults with pediatric-onset SCI. Moreover, given the established relation between appraisals and future outcomes, scores on the ADAPSS-sf will be examined in relation to patient demographics, injury characteristics, and secondary health and psychosocial outcomes within this population. No study thus far has examined the ADAPSS-sf within a pediatric-onset group, and replication of findings within this population would support its use within a variety of SCI populations. First, it was anticipated that the ADAPSS-sf would demonstrate good psychometric properties, including adequate internal consistency and 1-year stability, as well as a two-factor structure (Hypothesis 1). Second, it was expected that the
ADAPSS-sf would demonstrate adequate divergent and convergent validity in comparison with a variety of psychosocial variables by being positively correlated with measures of anxiety, depression, and post-traumatic stress, and negatively correlated with measures of life satisfaction, post-traumatic growth, and happiness (Hypothesis 2). Finally, it was predicted that negative cognitive appraisals would be associated with increased sleep difficulties, pain, urinary tract infections, pressure injuries, and health-related quality of life (Hypothesis 3 ). No study to date has examined the relation between the ADAPSS-sf and these secondary health outcomes, which is a notable gap considering the link between SCI and health outcomes and the potential role of cognitive appraisals in this relationship.

\section{Methods}

\section{Participants and procedures}

Participants were individuals who sustained an SCI at 18 years of age or younger, were 19 years of age or older at time of interview and were part of a larger longitudinal study of former patients at one of the three pediatric SCI programs (Shriners Hospitals for Children Chicago, Philadelphia and Northern California). See Table 1 for demographic information. Recruitment of participants commences as soon as the patients turn 19 years of age and present to clinic. Participants spoke English and did not have a significant brain injury or cognitive limitations based on medical report. Informed consent was obtained, and annual in-person or phone interviews were completed. Interviews last $\sim 1 \mathrm{~h}$. Injury-related data including neurological level was collected using hospital medical records. This study was approved by the institutional review board and is registered in a public access database at www. clinicaltrialsgov. Of the 490 participants enrolled in the longitudinal study, 115 (23\%) completed the cognitive appraisals measure using the ADAPSS-sf as this measure was added more recently to the ongoing project and is now administered annually to participants. An independent-samples $t$-test revealed no differences between the subsample of 115 and the overall sample in terms of gender, race, SCI etiology, level of injury, or injury severity. Significant mean differences were revealed in the age of injury of the subsample $(M=13.39$, $\mathrm{SD}=4.92)$ versus the overall sample $(M=14.58, \mathrm{SD}=$ $5.04) ; t(489)=2.66, p<0.05$, as well as the age of interview between subsample $(M=37.44, \mathrm{SD}=7.85)$ and the overall sample $(M=31.61, \mathrm{SD}=6.45) ; t(489)=$ $-8.79, p<0.001$. All analyses for the present study were completed using SPSS 22 [18]. 
Table 1 Participant demographic variables, injury characteristics, and ADAPSS-sf scores

\begin{tabular}{|c|c|c|c|c|}
\hline Characteristics & $N$ & Mean (SD) & $\%$ & $\begin{array}{l}\text { ADAPSS-sf } \\
\text { mean (SD) }\end{array}$ \\
\hline Age & 115 & $37.44(7.85)$ & & \\
\hline Age at injury & 115 & $13.39(4.92)$ & & \\
\hline Sex & 115 & & & \\
\hline Male & 67 & & 58.3 & $13.69(5.95)$ \\
\hline Female & 48 & & 41.7 & $14.29(6.35)$ \\
\hline Race & 115 & & & \\
\hline Caucasian & 97 & & 84.4 & $13.64(6.21)$ \\
\hline Any other race & 18 & & 15.7 & $15.56(5.32)$ \\
\hline Etiology & 115 & & & \\
\hline Vehicular/pedestrian & 61 & & 53.0 & $13.82(6.34)$ \\
\hline Violence & 5 & & 4.3 & $9.80(4.09)$ \\
\hline Fall/flying object & 12 & & 10.4 & $14.33(8.40)$ \\
\hline Sports & 22 & & 19.1 & $14.68(4.62)$ \\
\hline Medical/surgical & 15 & & 13.0 & $14.40(5.55)$ \\
\hline AIS injury severity ${ }^{a}$ & 109 & & & \\
\hline A & 74 & & 67.9 & $13.68(5.69)$ \\
\hline B & 10 & & 9.2 & $14.80(7.63)$ \\
\hline $\mathrm{C}$ & 13 & & 11.9 & $14.15(6.54)$ \\
\hline $\mathrm{D}$ & 12 & & 11.0 & $13.42(8.11)$ \\
\hline Level of injury & 115 & & & \\
\hline Tetraplegia & 58 & & 50.4 & $14.42(5.66)$ \\
\hline Paraplegia & 57 & & 49.6 & $13.47(6.52)$ \\
\hline Complete/incomplete & 109 & & & \\
\hline Complete & 74 & & 67.9 & $13.68(5.69)$ \\
\hline Incomplete & 35 & & 32.1 & $14.09(7.22)$ \\
\hline
\end{tabular}

${ }^{\mathrm{a}} \mathrm{A}=$ complete lack of motor and sensory function below the level of injury, $\mathrm{B}=$ some sensation below the level of injury, $\mathrm{C}=$ some muscle movement is spared below the level of injury, and $\mathrm{D}=$ majority of the muscles that are spared below the level of injury are able to move against injury

\section{Measures}

A structured questionnaire designed for this study was used to collect demographic, injury, and medical characteristics. Injury-related characteristics (age, severity and level of injury, and etiology) were obtained through the hospital system's SCI database and self-report (pressure injuries and urinary tract infections over the past year). Severity (American Spinal Injury Association Impairment Scale; AIS) and level of injury were based on the International Standards for Neurological Classification of Spinal Cord Injury [19].

\section{Appraisals of disability}

Self-reported appraisals of disability were measured using The Appraisals of DisAbility Primary and Secondary ScaleShort Form (ADAPSS-sf) [16]. The ADAPSS-sf is a self- report scale consisting of six domains that measure current appraisals of SCI. The three negative appraisals include Fearful Despondency ("Since my injury life is more frightening to me"), Overwhelming Disbelief ("I cannot believe that this has happened to me"), and Negative Perceptions ("I am going to miss out on so many aspects of my life"). Three-positive appraisals are represented by Growth and Resilience ("This experience has made me a stronger person"), Determined Resolve ("I will continue to live my life to its full capacity"), and Personal Agency ("There are many things I can do to change my situation"). Scores range from 1 (strongly disagree) to 6 (strongly agree). Higher levels of negative appraisals and lower levels of positive appraisals represent likelihood to appraise injury in terms of loss and threat and as unmanageable. Clinical cutoff scores will be calculated by statistical analysis with the current sample.

\section{Anxiety}

Symptoms of anxiety were assessed using the Beck Anxiety Inventory (BAI) [20].

\section{Depressive symptoms}

The Patient Health Questionnaire-9 (PHQ-9) [21] was used to determine symptoms of depression.

\section{Subjective global life satisfaction}

Life satisfaction was assessed with the Satisfaction with Life scale (SWLS) [22].

\section{Happiness}

Subject's perception of general happiness was measured using the General Happiness Scale [23].

\section{Post-traumatic growth}

The post-traumatic growth inventory was used to capture PTG in participants who sustained an SCI after 6 years of age [24].

\section{Post-traumatic stress symptoms}

The PTSD Checklist-Civilian Version [25, 26] was used to measure post-traumatic stress symptoms in the sample.

\section{Sleep}

Self-reported quality and patterns of sleep was assessed using the Pittsburgh Sleep Quality Index (PSQI) [27]. The 
PSQI measures seven domains of sleep and provides a global score of sleep functioning.

\section{Pain}

Six items from a range of pain questionnaires examined pain. Pain intensity (currently and in general), was assessed using an 11-point numerical rating scale $(0=$ no pain to $10=$ very intense pain) [28]. Pain frequency was rated on a six-point scale $(1=$ less than once per month to $6=$ daily $)$ [29] as was pain duration $(1=$ a few minutes to $6=$ several days/nights) [29]. Participants also responded to an item related to daily activity interference related to pain using a five-point scale $(1=$ no interference to $5=$ extreme interference). Distress related to pain was assessed using a fivepoint $(1=$ not at all/no suffering to $5=$ extremely bothered/ extreme suffering) [30].

\section{Health-related quality of life}

Health-related quality of life was measured using the 12item Short-Form Health Survey, Version 2 (SF-12v2 Health Survey [31]).

\section{Results}

\section{ADAPSS-sf factor structure and psychometric properties (Hypothesis 1)}

Participant characteristics and mean ADAPSS-sf scores are presented in Table 1. The total score range on the ADAPSSsf was 6 to 36, with participants endorsing the full range of individual item scores. The mean for the full sample $(N=$ $115)$ was $13.94(\mathrm{SD}=6.10)$. Using the standardization and norming procedures previously employed for this measure using an acute SCI sample with the $85^{\text {th }}$ percentile categorized as significant [17], clinical cutoff scores were also calculated. For the total ADAPSS-sf, participants that scored 22 or more may be categorized as significant. Thus, a score of 22 or more on the ADAPSS-sf may represent clinically elevated maladaptive appraisals within the pediatric-onset adult SCI population.

Preliminary analyses revealed that the Kaiser MeyerOlkin measure meritoriously verified the suitability of the data for factor analysis $(0.80)$. Bartlett's test of sphericity, $\chi^{2}$ $(15, N=115)=169.62, p<0.001$ and the determinant (0.22) indicated that the correlations between items were sufficiently large, warranting principal component factor analysis. The amount of variance across items in extracted communalities ranged from 0.23 (Item 6) to 0.62 (Item 4). Inconsistent with prior findings, which revealed two principal components of Catastrophic Negativity and Determined Resilience [10, 12, 17], only one factor was extracted. A one-component structure was supported through the scree test and eigenvalue above 1 criterion, with a two-factor structure containing one factor eigenvalue at 0.887 .

The Cronbach's alpha coefficient for the six-item scale was good $(\alpha=0.74 ; N=115)$, indicating adequate reliability. Deleting Item 6 ("There are many things I can do to change my situation") would only slightly improve internal consistency $(\alpha=0.75)$, which was avoided due to theoretical foundations outlined by Dean and Kennedy [16]. Corrected item-total correlations ranged from .34 (Item 6) to 0.64 (Item 4), which reveals adequate item discrimination. The Pearson's correlation coefficient average between items was 0.36. Table 2 presents the means, standard deviations, and inter-item Pearson's correlations for the ADAPSS-sf total score and individual items.

Test-retest reliability for the ADAPSS-sf was measured using Pearson correlation among a subset of 30 participants (of the original 115) that had taken two consecutive annual evaluations. Using Evans [32] verbal definitions of strength of correlations $(0.00-0.19=$ "very weak," $0.20-0.39=$ "weak," $0.40-0.59=$ "moderate," $0.60-0.79=$ "strong,"

Table 2 Means, standard deviations, cutoff score, and inter-item Pearson's correlations for the ADAPSS-sf

\begin{tabular}{llllllllll}
\hline Variable & $M$ & SD & TS & 1 & 2 & 3 & 4 & 5 \\
\hline Total score & 13.94 & 6.10 & -- & & & & \\
Items & & & & & & \\
1. Since my injury life is more frightening for me. & 2.47 & 1.69 & $0.77^{* * *}$ & -- & & & \\
2. I cannot believe that this has happened to me. & 2.41 & 1.75 & $0.69^{* * *}$ & $0.38^{* * *}$ & -- & & \\
3. I will continue to live my life to its full capacity. & 1.31 & 0.69 & $0.66^{* * *}$ & $0.46^{* * *}$ & $0.39^{* * *}$ & -- \\
4. I am going to miss out on so many aspects of my life & 2.97 & 1.78 & $0.79^{* * *}$ & $0.61^{* * *}$ & $0.45^{* * *}$ & $0.44^{* * *}$ & -- \\
5. This experience has made me a stronger person. & 1.82 & 1.23 & $0.59^{* * *}$ & $0.37^{* * *}$ & $0.23^{*}$ & $0.52^{* * *}$ & $0.36^{* * *}$ & -- \\
6. There are many things that I can do to change my situation. & 2.97 & 1.75 & $0.58^{* * *}$ & $0.28^{* *}$ & $0.25^{* *}$ & $0.25^{* *}$ & $0.28^{* *}$ & 0.18 & -- \\
\hline
\end{tabular}

$N=115$ for all variables

${ }^{*} p<0.05 .{ }^{* *} p<0.01$. ${ }^{* * *} p<0.001$ 
$0.80-1.0=$ "very strong") the 1 -year stability for the total score was strong $(r=0.81, p<0.001)$. Correlations were positive, significant, and strong for Items $1,2,4$, and 6 ( $r=$ $0.55-0.80, p<0.01)$, positive, significant, and moderate for Item $3(r=0.33, p=0.02)$, and only trending significance for Item 5 ( $r=0.33, p=0.07)$. A paired-samples $t$-test for the total score revealed a significant mean difference in the scores between the first administration $(M=12.53, \mathrm{SD}=$ 4.57) and the second $(M=15.13, \mathrm{SD}=5.06) ; t(29)=$ -4.69, $p<0.001, d=0.54$, representing a medium effect size. Paired-samples $t$-tests for the six items demonstrated that there were higher means across time in Item $1[t(28)=$ $-3.36, p=0.001]$ and Item $2[t(29)=-2.28, p=0.05]$, and a lower mean for Item $6[t(29)=-2.52, p<0.05]$.

\section{Association of ADAPSS-sf with patient demographics and injury characteristics}

In order to inform generalizability and understanding of the ADAPSS-sf across participants, a series of exploratory analyses were conducted examining the association between participant demographics and injury characteristics. No significant differences on the ADAPSS-sf emerged between male and female participants, $t(113)=-0.52, p=0.228$. There were also no significant differences between participants identifying as Caucasian and racial minority participants, $t(113)=-1.23, p=0.425$. Similar to findings of prior studies of the ADAPSS and the ADAPSS-sf [10, 16], but in contrast with another [12], current age was not significantly correlated with the ADAPSS-sf total score or individual items. The age of injury demonstrated a positive and small, yet significant, association with the ADAPSS-sf total score and Items 2 and 4 (see Table 3). This suggests that individuals who sustained SCI at older ages are more likely to endorse negative cognitive appraisals of their SCI.

Group differences on the ADAPSS-sf total score for level of injury, severity, and etiology were examined with a series of one-way ANOVAs or paired-samples $t$-tests. In contrast with prior studies using veteran samples [10, 12], there were no differences between groups in terms of etiology (vehicular/pedestrian, violence, fall/flying object, sports, medical/surgical). Similarly, there were no group differences in level of injury (tetraplegia, paraplegia), completeness of injury (complete, incomplete), or injury severity, partially supporting the findings of previous research.

\section{Association of ADAPSS-sf with psychosocial functioning (Hypothesis 2)}

Pearson correlations were conducted in order to measure the association between the ADAPSS-sf total score and various psychosocial outcomes (see Table 4). The correlations
Table 3 Pearson's correlations of participant age and age at injury with ADAPSS-sf items and total score

\begin{tabular}{lll}
\hline & \multicolumn{2}{l}{ Correlations } \\
\cline { 2 - 3 } Variable & Age & Age at injury \\
\hline ADAPSS-sf TS & -0.00 & $0.27^{* *}$ \\
Items & & \\
Item 1 & -0.04 & 0.14 \\
Item 2 & -0.02 & $0.21^{*}$ \\
Item 3 & 0.01 & 0.15 \\
Item 4 & 0.07 & $0.34^{* * *}$ \\
Item 5 & 0.00 & 0.15 \\
Item 6 & -0.03 & 0.07 \\
\hline
\end{tabular}

Age and age at injury are measured on a continuous scale. $N=115$ for all variables

${ }^{*} p<0.05 .{ }^{* *} p<0.01{ }^{* * *} p<0.001$

${ }^{\text {a }}$ Reverse-coded

Table 4 Pearson's correlations of psychosocial and secondary health outcomes with ADAPSS-sf total score

\begin{tabular}{lc}
\hline Variable & $\begin{array}{l}\text { Correlations } \\
\text { ADAPSS-sf TS }\end{array}$ \\
\hline BAI & $0.48^{* * *}$ \\
PHQ-9 & $0.46^{* * *}$ \\
PCL-C & $0.39^{* *}$ \\
GHS & $-0.68^{* * *}$ \\
PTGI & $-0.32^{* *}$ \\
SWLS & $-0.67^{* * *}$ \\
PSQI & $0.19^{*}$ \\
SF-12v2 & $0.38^{* * *}$ \\
UTI Frequency & 0.02 \\
Pressure injury frequency & $0.26^{* *}$ \\
Pain intensity (average) & 0.13 \\
Current pain & $0.12^{*}$ \\
Pain frequency & 0.13 \\
Pain duration & 0.01 \\
Distress from pain & $0.21^{*}$ \\
Activity interference from pain & 0.10 \\
\hline
\end{tabular}

BAI Beck Anxiety Inventory total score, $P H Q$ Patient Health Questionnaire-9 depression total score, $P C L-C$ PTSD ChecklistCivilian total score, GHS General Happiness Scale total score, PTG Post-traumatic Growth Inventory total score, SWLS Satisfaction with Life total score, PSQI Pittsburgh Sleep Quality Index total score, SF$12 v 2$ Medical Outcomes Study Short Form version 2, UTI urinary tract infection

${ }^{*} p<.05 .{ }^{* *} p<.01{ }^{* * *} p<.001$

${ }^{\mathrm{a}} n=65$

${ }^{\mathrm{b}} n=95$

between the ADAPSS-sf total score and anxiety, depression, and post-traumatic stress were positive, significant, and moderate to strong. That is, as mental health concerns 
increased, negative SCI-related appraisals increased as well. The correlations between the ADAPSS-sf total score and other measures of psychosocial well-being, such as satisfaction with life, happiness, and post-traumatic growth were negative, significant, and moderate to strong. Thus, when these measures of psychosocial well-being were rated as higher by participants, negative SCI-related appraisals were lower.

\section{Association of ADAPSS-sf with secondary health outcomes (Hypothesis 3)}

In order to test the association between various secondary health outcomes and the ADAPSS-sf total score, Pearson correlations were computed and are presented in Table 4. The correlation between the ADAPSS-sf and subjective perception of health was significant, positive, and moderate. Significant, positive, and small correlations were revealed between the ADAPSS-sf total score and number of pressure injuries, pain, distress related to pain, and sleep difficulties. No relation was found between the ADAPSS-sf total score and urinary tract infections, or for subjective ratings of pain frequency, intensity, duration, or activity interference owing to pain.

\section{Discussion}

The current study is the first to examine the psychometric properties of a brief measure of cognitive appraisals, the ADAPSS-sf, in adults with pediatric-onset SCI. Furthermore, the relation between this six-item instrument and patient characteristics, psychosocial outcomes, and secondary health outcomes was investigated.

Study findings support sound psychometrics of the ADAPSS-sf in an adult population who had sustained a pediatric-onset SCI, which further strengthens the utility of the instrument across a variety of SCI populations. The measure demonstrated strong test-retest reliability over a 1year period and internal consistency that was well within the target range for a broad construct as recommended in the literature [33]. Inconsistent with the three prior studies examining the ADAPSS-sf $[10,12,17]$, only one component factor was revealed as opposed to a two-factor structure of Catastrophic Negativity and Determined Resilience [16], suggesting that the ADAPSS-sf may instead serve as a unitary construct. However, it should be noted that the three previously cited studies had a larger sample size and thus further study is needed in order to confirm or establish a factor structure within this population. Moreover, the full range of the ADAPSS-sf scores was endorsed, which is evidence of the scale's sensitivity to measuring variability in SCI-related appraisals. In addition to sound psychometrics, the six-item ADAPSS-sf is notable for its brevity, reducing patient burden contrasted with the full 36item form.

Exploratory analyses were conducted in order to determine differences in SCI-related appraisals on the ADAPSSsf based on demographic and injury characteristics in order to inform generalizability, as well as to identify potential vulnerable groups. There were no differences related to current age, gender, or race. In contrast to prior studies using veteran samples $[10,12]$, there were no differences between groups in terms of SCI etiology, nor were there differences in injury severity or level. It should be noted that subgroup sizes and imbalances may affect the power needed to adequately identify differences across demographics and injury characteristics and thus further research with larger samples may be useful. Importantly, current findings indicate that individuals who sustained SCI at an older age may be more likely to endorse negative appraisals of their SCI. Taken together, these results suggest that appraisals may differ for a pediatric-onset adult sample versus a veteran sample, and those sustaining SCI during adolescence may require increased intervention and a greater emphasis in the literature. Previous research has demonstrated that individuals who sustain injury later in life are at increased risk for anxiety, depression, reduced life satisfaction, and diminished reliance on adaptive coping strategies [34]. Current results seem to support these findings even within adults with pediatric-onset, suggesting that later injuries may lead to increased adjustment difficulties and increased negative SCI-related appraisals.

As anticipated, the current study results suggest that higher negative SCI-related appraisals was related to higher mental health difficulties (Hypothesis 2), both confirming and expanding on previous literature using the ADAPSS-sf. Consistent with prior studies [10, 12, 17], the ADAPSS-sf demonstrated convergent validity by being positively and significantly associated with anxiety, depression, and satisfaction with life. Extending beyond previous literature, the current study revealed positive associations with posttraumatic stress symptoms and negative associations with post-traumatic growth and general happiness, further lending evidence in support of the measure's convergent validity. While causality cannot be determined in this study, current findings suggest that individuals with appraisals of disbelief, loss, and fear in the absence of the protective appraisals of growth and personal agency are at greater risk for the development of psychopathology and reduced life satisfaction and happiness following SCI. Thus, assessment of cognitive appraisals, and perhaps cognitive distortions [35] as well as perceived manageability [36], may be important in identifying individuals experiencing difficulties with emotional adjustment following SCI. Furthermore, negative cognitive appraisals may be targeted during 
intervention using cognitive behavioral therapy (CBT)based coping effectiveness training approaches in order to improve emotional and medical outcomes for these individuals $[37,38]$. While responses on the ADAPSS-sf were heterogenous, it is also important to note that a sizable percentage of participants in the study employed positive and adaptive appraisals, underscoring the tendency for resilience following SCI.

Another novel contribution to a notable gap in literature was the finding that cognitive appraisals measured by the ADAPSS-sf are associated with a variety of secondary health outcomes (Hypothesis 3). Negative SCI-related appraisals were associated with sleep difficulties, pressure injuries, current pain, distress from pain, and poor overall subjective ratings of health. Again, while clinical significance is not determined, these findings support the clinical utility of the ADAPSS-sf in identifying both physical and emotional health following SCI. Previous research has revealed that elevated depression and anxiety symptoms are predictive of several SCI-related health difficulties [39]. The ADAPSS-sf provides a useful tool to improve understanding of how appraisals may influence and interact with physical health and medical treatment adherence for this population. The determination of a clinical cutoff score for this measure among this population will further improve its utility in identifying those at risk for clinically elevated negative appraisals.

\section{Study limitations}

The findings of the current study should be interpreted in the context of methodical and sampling limitations. The cross-sectional design of the study precludes our ability to identify causal relationships between the ADAPSS-sf and various outcomes. Adjustment to a disability is a transactional process rather than a singular event, thus future research would benefit from longitudinal study to test SCI-related appraisals and their relation to psychosocial and secondary health outcomes over time. The majority of participants were Caucasian, and all participants had previously received care from a single pediatric specialty hospital system, which may limit the generalizability of the findings to other ethnicities and those who received care from different facilities. Nevertheless, the three SCI organizations serve a wide network of regions across the United States (Chicago, Philadelphia and Northern California) and represent individuals of varying socioeconomic status. Moreover, a sample of individuals that have visited a hospital and participated in a longitudinal study over several years may be distinct from the general pediatric-onset SCI population and potentially biased toward positive appraisals. While beyond the scope of the current study, future research is needed to further understand the development and course of SCI-related appraisals, as well clinical interventions that may target specific appraisals in order to improve emotional and physical health and well-being.

\section{Conclusions}

Using reliable and valid measures to track cognitive appraisals following SCI is critical given the link between appraisals and recovery. By demonstrating good internal validity, test-retest reliability, convergent and face validity, and brevity, this study confirms the use of the ADAPSS-sf in a pediatric-onset population, bolstering its use across a variety of SCI populations in both clinical and research settings. Moreover, the current study revealed that such appraisals are associated with both psychosocial and secondary health outcomes, further supporting the ADAPSS-sf as a valuable tool for clinicians and researchers across disciplines.

\section{Data archiving}

The datasets generated analyzed during the current study are not publicly available given the ongoing nature of the hospital-wide study involving both current and former patients but are available from the authors upon reasonable request with permission from Shriners Hospitals for Children.

Acknowledgements This paper is dedicated to the memory of our esteemed colleague and collaborator, Professor Paul Kennedy.

Funding This work was funded by Craig $\mathrm{H}$. Neilsen Foundation, grant \# 324671, and Shriners Hospitals for Children-Chicago.

Author contributions KD was responsible for analyzing data, interpreting results, and writing the manuscript. $\mathrm{KZ}$ was responsible for collecting data, drafting methods, and providing feedback on written manuscript. KC drafted methods, assisted with data analysis, and provided feedback on written manuscript. LV assisted with study design, data collection and interpretation of results, and providing feedback on the written manuscript.

\section{Compliance with ethical standards}

Conflict of interest The authors declare that they have no conflict of interest.

Statement of ethics This study was approved by the institutional review board and is registered in a public access database at www. clinicaltrialsgov. We certify that all applicable institutional and governmental regulations concerning the ethical use of human volunteers were followed during the course of this research.

Publisher's note Springer Nature remains neutral with regard to jurisdictional claims in published maps and institutional affiliations. 


\section{References}

1. Zebracki K, Vogel, L. Epidemiology of childhood-onset spinal cord injuries in the United States. In: Rahimi-Movaghar V, Jazayeri S, Vaccaro A, editors. Epidemiology of spinal cord injuries. Hauppauge, NY: Nova Science Publishers Inc.; 2012.

2. Goodridge D, Rogers M, Klassen L, Jeffery B, Knox K, Rohatinsky N, et al. Access to health and support services: perspectives of people living with a long-term traumatic spinal cord injury in rural and urban areas. Disabil Rehabil. 2015;8288:1-10.

3. Hwang M, Chlan KM, Vogel LC, Zebracki K. Substance use in young adults with pediatric-onset spinal cord injury. Spinal Cord. 2012;50:497-501.

4. January A, Zebracki K, Chlan K, Vogel LC. Sleep, well-being, and psychological symptoms in adults with pediatric-onset spinal cord injury. Rehabil Psychol. 2015;60:328-34.

5. January A, Zebracki K, Chlan K, Vogel L. Symptoms of depression over time in adults with pediatric-onset spinal cord injury. Arch Phys Med Rehab. 2014;95:109-15.

6. Chen Y, Anderson C, Vogel L, Chlan K, Betz R, McDonald C. Change in life satisfaction of adults with pediatric-onset spinal cord injury. Arch Phys Med Rehabilitation. 2008;89:2285-92.

7. Chevalier Z, Kennedy P, Sherlock O. Spinal cord injury, coping, and psychological adjustment: a literature review. Spinal Cord. 2009;47:778-82.

8. Williams R, Murray A. Prevalence of depression after spinal cord injury: a meta-analysis. Arch Phys Med Rehab. 2015;96:133-40.

9. Peter C, Müller R, Post MW, van Leeuwen CM, Werner CS, Geyh S. Psychological resources, appraisals, and coping and their relationship to participation in spinal cord injury: a path analysis. Arch Phys Med Rehab. 2014;95:1662-71.

10. Mignogna J, Christie A, Holmes S, Ames H, Wegener S. Measuring disability-associated appraisals for veterans with spinal cord injury. Rehabil Psychol. 2015;60:99-104.

11. Kennedy P, Kilvert A, Hasson L, Wegener S. A 21-year longitudinal analysis of impact, coping, and appraisals following spinal cord injury. Rehabil Psychol. 2016;61:92-101.

12. McDonald SD, Goldberg-Looney LD, Mickens MN, Ellwood M, Mutchler BJ, Perrin PB. Appraisals of DisAbility Primary and Secondary Scale-Short Form (ADAPSS-sf): psychometrics and association with mental health among U.S. military veterans with spinal cord injury. Rehabil Psychol. 2018;63:372-38.

13. Lazarus RS, Folkman S. Stress, appraisal and coping. New York, NY: Springer; 1984.

14. Duff J, Kennedy P. Spinal cord injury. In: Llewelyn SP, Kennedy $\mathrm{P}$, (editors.) Handbook of clinical health psychology. Chichester, UK: John Wiley and Sons; 2003. p. 251-78.

15. Kennedy P, Evans M, Sandhu N. Psychological adjustment to spinal cord injury The contribution of coping, hope, and cognitive appraisals. Psychol Health Med. 2009;14:17-33.

16. Dean R, Kennedy P. Measuring appraisals following acquired spinal cord injury: a preliminary psychometric analysis of the appraisals of disability. Rehabil Psychol. 2009;54:222-31.

17. Eaton R, Jones K, Duff J. Cognitive appraisals and emotional status following a spinal cord injury in post-acute rehabilitation. Spinal Cord. 2018;56:1151-7.

18. IBM Corp. IBM SPSS Statistics for Windows, Version 22.0. Armonk, NY: IBM Corp; 2013.

19. Kirshblum SC, Burns SP, Biering-Sorensen F, Donovan W, Graves DE, Jha A, et al. International standards for neurological classification of spinal cord injury. J Spinal Cord Med. 2011;34: 535-46.

20. Beck AT, Steer RA. Beck anxiety inventory manual. San Antonio, TX: Psychological Corporation; 1993.

21. Kroenke K, Spitzer RL, Williams JBW. The PHQ-9: Validity of a brief depression severity measure. J Gen Intern Med. 2001;16: 606-13.

22. Diener E, Emmons R, Larsen J, Griffin S. The satisfaction with life scale. J Pers Assess. 1985;49:71-5.

23. Lyubomirsky S, Lepper H. A measure of subjective happiness: preliminary reliability and construct validation. Soc Indic Res. 1999;46:137-55.

24. Tedeschi RG, Calhoun LG. The posttraumatic growth inventory: measuring the positive legacy of trauma. J Trauma Stress. 1996;9:455-71.

25. Blanchard EB, Jones-Alexander J, Buckley TC, Forneris CA. Psychometric properties of the PTSD checklist (PCL). Behav Res Ther. 1996;34:669-73.

26. Ruggiero KJ, Ben KD, Scotti JR, Rabalais AE. Psychometric properties of the PTSD checklist-civilian version. J Trauma Stress. 2003;16:495-502.

27. Buysse DJ, Reynolds CF III., Monk TH, Berman SR, Kupfer DJ. The Pittsburgh sleep quality index: a new instrument for psychiatric practice and research. Psychiatry Res. 1989;28: 193-213.

28. von Baeyer CL, Spagrud LJ, McCormick JC, Choo E, Neville K. Three new datasets supporting use of the Numerial Rating Scale (NRS-11) for children's self-reports of pain intensity. Pain. 2009; 143:223-7.

29. Palermo TM, Valenzuela D, Stork P. A randomized trial of electronic versus paper pain diaries in children: Impact on compliance, accuracy, and acceptability. Pain. 2004;107:213-9.

30. McGrath PA. Pain in children: Nature, assessment, and treatment. New York: Guilford Press; 1990.

31. Ware JE, Kosinski M, Keller SD. A 12-Item Short-Form Health Survey: construction of scales and preliminary tests of reliability and validity. Med Care. 1996;34:220-33.

32. Evans JD. Straightforward statistics for the behavioral sciences. Pacific Grove, CA: Brooks/Cole Publishing; 1996.

33. Clark LA, Watson D. Constructing validity: Basic issues in objective scale development. Psychol Assess. 1995;7:309-19.

34. Geyh S, Kunz S, Müller R, Peter C. Describing functioning and health after spinal cord injury in the light of psychological-personal factors. J Rehabil Med. 2016;48:219-34.

35. Pollard C, Kennedy P. A longitudinal analysis of emotional impact, coping strategies and post-traumatic psychological growth following spinal cord injury: a 10-year review. Brit J Health Psych. 2007;12:347-62.

36. Kennedy P, Scott-Wilson U, Navtej S. The psychometric analysis of a brief and sensitive measure of perceived manageability. Psychol Health Med. 2009;14:454-65.

37. King C, Kennedy P. Coping effectiveness training for people with spinal cord injury: Preliminary results of a controlled trial. Br J Clin Psychol. 1999;38:5-14.

38. Kennedy P, Duff J, Evans M, Beedie A. Coping effectiveness training reduces depression and anxiety following traumatic spinal cord injuries. Br J Clin Psychol. 2003;42:41-52.

39. January A, Zebracki K, Chlan K, Vogel L. Mental health and risk of secondary medical complications in adults with pediatric-onset spinal cord injury. Top Spinal Cord Inh Rehabil. 2014;20:1-12. 